\title{
Harmonic Rejection in 2.4 GHz Gilbert Cell Mixer for Terrestrial Microwave Communication systems
}

\author{
R. Venkatesan \\ Post graduate scholar \\ SSN College of Engineering \\ Kalavakkam, Chennai-603110
}

\author{
K.J. Jegadish Kumar \\ Associate Professor \\ SSN College of Engineering, \\ Kalavakkam, Chennai-603110
}

\begin{abstract}
Mixer is one of the significant components of the radio frequency transceiver that translate baseband signal into RF and vice versa. In this paper, $2.4 \mathrm{GHz}$ double balanced Gilbert Cell Mixer topology was simulated to study its important properties such as Conversion Gain, Linearity, Noise Figure and Port Isolation. Further, a band pass filter is added in to the design at IF port so as to reject harmonics. The mixer topology is designed and simulated using NMOS $180 \mathrm{~nm}$ CMOS technology in Advanced Design Systems. As a result, conversion gain of $8.303 \mathrm{~dB}$, high linearity, high port isolation and Harmonic Rejection Ratio $>80 \mathrm{~dB}$ is achieved.
\end{abstract}

\section{General Terms}

Harmonic rejection in down conversion Gilbert cell Mixer

\section{Keywords}

Gilbert Cell Mixer, Conversion gain, linearity, Harmonic rejections

\section{INTRODUCTION}

In recent years, Radio Frequency (RF) circuitry has been significant in design of reliable and efficient (RF) communicationcircuits, which takes great strides from cell phones to base stations. The most significant core of the RF circuitry is mixer in transmitter and receiver.

Mixer is a electronic RF circuit that has two inputs and one output. It mixes two signals, RF or intermediate frequency (IF) signal with another input signal from local oscillator to generate either IF or RF to assist as down conversion in receiver or upcoversion in transmitter.

The ideal nonlinear mixer performs heterodyning process and is explained mathematically in equations (1) (2) and (3). In the following equations, which were obtained and are summarized from [1].

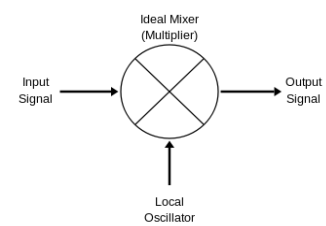

Fig 1:Down Conversion Mixer Basic Circuit

If both input signals show:

$$
\begin{aligned}
& a=A \cos w_{1} t \\
& b=B \cos w_{2} t
\end{aligned}
$$

The mixer output signal after mixing yields, $a \cdot b=A \cos w_{1} t \cdot B \cos w_{2} t=\frac{A B}{2} \cos \left(w_{1}-w_{2}\right) t+\frac{A B}{2} \cos \left(w_{1}+w_{2}\right) t$

Thus, the mixer output consists of sum and difference of the two input signals. Later a filter is used to filter out the undesired frequency and pass desired frequency. The chosen function, can be either the differences or summation of the input frequencies,receives the term down conversion mixer or up conversion mixer respectively.

Typically mixer designs are specified with primary definitions that are defined and explained below: Conversion Gain (CG), Linearity, Noise Figure (NF), and port isolations. The CG is a ratio between the output signal and the input signal. Itis usually measured in decibels $(\mathrm{dB})$ or, milli-decibels $(\mathrm{dBm})$. The linearity of the mixer is defined as how well the mixer reacts to the mixing of frequencies. NF is a ratio of the signalto-noise ratio (SNR) at the input port and the SNR at the out port. Finally, the port isolation parameter show how much leakage of signal occurs between two ports is avoided.

Down conversion mixer has two input, one is the RF input signal, another is the local oscillator signal. The local oscillator input signal $\mathrm{W}_{\mathrm{LO}}$ is the wanted signal the additional local oscillator frequency called harmonics, generated along the fundamentals frequency $\mathrm{W}_{\mathrm{LO}}$ are unwanted In this work, to design down conversion mixer with harmonics rejection technique using a simple band pass filter with the center pass frequency of $250 \mathrm{MHzIn}$ section three, the double balance (DB) Gilbert cell mixer design is introduced and explained using analytical equations. Section Four explains the implementation and simulation of double balance (DB) Gilbert cell design. Finally, in section five overall work is summarized and concluded.

\section{RELATED WORK}

Kuei-Cheng Lin et al. (2013) [2] proposed a 4.2-mW 6-dB gain $5-65-\mathrm{GHz}$ gate-pumped down-conversion mixer using Darlington cell for $60-\mathrm{GHz}$ CMOS receiver. The mixer is implemented in a standard 90-nm CMOS process under $1-\mathrm{V}$ supply voltage. The measured mixer achieves a $6.5 \mathrm{~dB}$ in maximum $\mathrm{CG}$, a $60-\mathrm{GHz}$ bandwidth in 3-dB bandwidth, and a $0.15 \mathrm{~mm}^{2}$ in chip size at a power consumption of $4.2 \mathrm{~mW}$.The receiver outperforms several recently published studies and will find extensive applications in $60-\mathrm{GHz}$ transceiver designs.

Hwann-Kaeo Chiou, Hung-Ting Chou. (2013) [3] proposed an ultra-low power V-band source -driven down conversion mixer with low-loss and broadband asymmetrical broadsidecoupled balun in 90-nm CMOS technology. The forward body biased techniques reduces the threshold voltage and supply voltage for operations in the near weak inversion region in millimeter-wave mixer designs. The proposed FBB mixer has 
an excellent, which is better than those of other recently developed MMW active down conversion mixers.

Ning Zhang et al. (2009) [4] proposed a W-band active down conversion mixer in bulk CMOS. A W-band active down conversion mixer has been demonstrated using low leakage (higher $\mathrm{V}_{\mathrm{T}}$ ) NMOS transistor of a $65-\mathrm{nm}$ digital CMOS process. It achieves higher conversion gain, low power. Digital 65-nm CMOS process is used for low cost consumer oriented applications.

Aslam A. Rafi, T.R Viswanathan. (2013) [5] proposed a harmonic rejection mixing techniques using clock-gating. The clock gate harmonic rejection mixing techniques address the challenges in a low-cost wideband receiver design and achieve significantly reduced sensitivity to mismatches in devices operating at high frequency. This technique is achieved for third, fifth, seventh, and ninth HR ratios.

Fujian Lin et al. (2014) [6] proposed a sine LO square law harmonic rejection mixer theory, implementation, and application. The square law harmonic rejection mixer driven by a sine local oscillator is proposed for wideband and tunable rejection of all LO harmonics. The key performance including the gain, noise figure, third order intercept point, and harmonic rejection ratio with respect to the amplitude and purity of the sine LO are analyze. SL-HRM incorporates a voltage boosted technique to surmount the gain and NF penalties of square law mixing.

Renzhi Liu et al. (2014) [7] proposed a wideband RF receiver with>80 $\mathrm{dB}$ harmonic rejection ratio. In their work, a wideband RF receiver with harmonic rejection is presented. Both gain mismatch and phase mismatch of the HR mixerhave calibrated independently using a digital calibration scheme. After calibration both the $3^{\text {rd }}$ order, $5^{\text {th }}$ order harmonic rejection ratio are greater than $80 \mathrm{dBm}$ and $70 \mathrm{dBm}$ respectively.

Huajiang Zhang et al. (2012) [8] proposed a harmonic rejection mixer with improved design algorithm for broadband TV tuners. A wide band harmonic rejection mixer for TV tuners with an improved design algorithm is fabricated in $65 \mathrm{~nm}$ CMOS process. A more relastic mathematic formula is derived to calculate harmonic rejection performance. The third and fifth order harmonic rejection ratio were calculated.

TakafumiYamaji et al. (2011) [9] proposed a harmonic signal rejection schemes of polyphase downconverters. The harmonic signal rejection capability and the rejection schemes of polyphase downconverter are discussed. An analysis of a simple model of a polyphase downconverter shows that nphase converter can reject the harmonic signal. Harmonic rejection with Polyphase downconverter uses two schemes, weighting and summing system and a partial common mode rejection system.

\section{GILBERT CELL MIXER \\ 3.1 Basics of Gilbert Cell Mixer}

The Gilbert cell mixer or Gilbert cell multiplier referred as four-quadrature multiplier is a form of RF mixer circuit that is widely used in integratedcircuitsto provide a high level of performance. For use of a Gilbert cell mixer within an integrated circuit, the choice type will depend to a large degree upon the process used for the IC manufacture.

Most widely used RF Gilbert cell mixer or Gilbert cell multiplier is a form of two differential transistor pair double balanced mixer that is able to exploit the symmetrical topology to remove the unwanted RF \& LO output signals from the IF by cancellation. As performance of adjacent components within an integrated circuit is likely to be well matched, the circuit will be well balanced and suppression of the unwanted signal components will be high.

\subsubsection{Propertiesof Gilbert Cell Mixer ConversionGain}

This is the ratio (in $\mathrm{dB}$ ) between the IF signal (usually the difference frequency between the RF and LO signals) and the RF signal

Conversion Gain $[\mathrm{dB}]=$ output IF power $[\mathrm{dBm}]-\mathrm{RF}$ input power $[\mathrm{dBm}]$ NoiseFigure

Noise figure is defined as the ratio of SNR at the IF port to the SNR of the RF port

\section{Isolation}

These parameter define how much signal leakage will occur between pairs of ports. (i.e) RF to LO, LO to IF and RF to IF. So if for example RF to IF isolation was specified at $35 \mathrm{~dB}$ this means that the RF at the IF port will be $35 \mathrm{~dB}$ lower than the RF applied to RF port.There are multiple types of isolation: LO-to-RF, LO-to-IF, RF-to-IF isolation.LO to RF port isolation is by far the biggest short coming.

\section{Linearity}

\section{(i) $1 \mathrm{~dB}$ Compression Point}

Like other non-resistive networks, a mixer is amplitudenonlinear above a certain input level resulting in a gain compression characteristic. Most mixers have the $1 \mathrm{~dB}$ compression point specified at the input (i.e) the single-tone input signal level at which the output of the mixer has fallen 1 $\mathrm{dB}$ below the expected output level. For typical double balanced mixers this figure is $6 \mathrm{~dB}$ below the $\mathrm{LO}$ power level.(so performance can be improved by overdriving the $\mathrm{LO}$ port)

\section{(ii) Intermodulation Performance}

This parameter is the same as specified for amplifiers and measured in a similar way. It measured by applying two closely spaced input tones at frequencies F1anLd F2. Third order products from the mixing of these tones with the $\mathrm{LO}$ (at frequency $\mathrm{F}_{\mathrm{LO}}$ ) occur at frequencies given by $\left(2 \mathrm{~F}_{1} \pm \mathrm{F}_{2}\right) \pm \mathrm{F}_{\mathrm{LO}}$ and $\left(2 \mathrm{~F}_{2} \pm \mathrm{F}_{1}\right) \pm \mathrm{F}_{\mathrm{LO}}$. In the case of the mixer, the third order products of most interest are $\left(2 \mathrm{~F}_{1}-\mathrm{F}_{2}\right)-\mathrm{F}_{\mathrm{LO}}$ and $\left(2 \mathrm{~F}_{2}-\mathrm{F}_{1}\right)-\mathrm{F}_{\mathrm{LO}}$ as they fall in, or close to the IF band. In a receiver, the most damaging distortion products are odd-order, since those are most likely fall within the same pass band as the desired signal. The highest amplitude will be the lowest order, (i.e)the $3^{\text {rd }}$ order products. The most common figure of merit for intermodulation distortion (IMD) is third order intercept (TOI).

Harmonic Rejection Ratio $=$ The ratio of amplitude of $3^{\text {rd }}$ harmonic without filter to the amplitude of $3^{\text {rd }}$ harmonic with filter

\subsection{Introduction to DB Gilbert Cell Mixer}

Double Gilbert cell mixer is the common active mixer architecture, which contains improvements over other designs including the DB Gilbert cell mixer.The DB mixer can achieve higher mixer specifications in the area of CG, linearity, and port isolation at the cost of a small increase in $\mathrm{NF}$ and overall designs power consumption. 
DB Gilbert Mixer basically two SBmixers placed together. Fig 2 illustrates the circuit of themixer [10]. Placing current source at the bottom of the circuit is called source degeneration and is used to aid linearity and stability of the mixer. This mixer circuit is modified from SB mixer by having the differential RF input in the transconductance stage with two transistor while the switching operations consist of another pair of transistor and making total of transistors.
Each time the switches change position the small signal current changes directions through the load resistors, and mixes with the LO signal, converting the current into a voltage. The differential output is then measured for gain and other mixer definitions. The designequations ofthe DB Gilbert cell mixer are almost similar to the SB mixer, but are not identical.

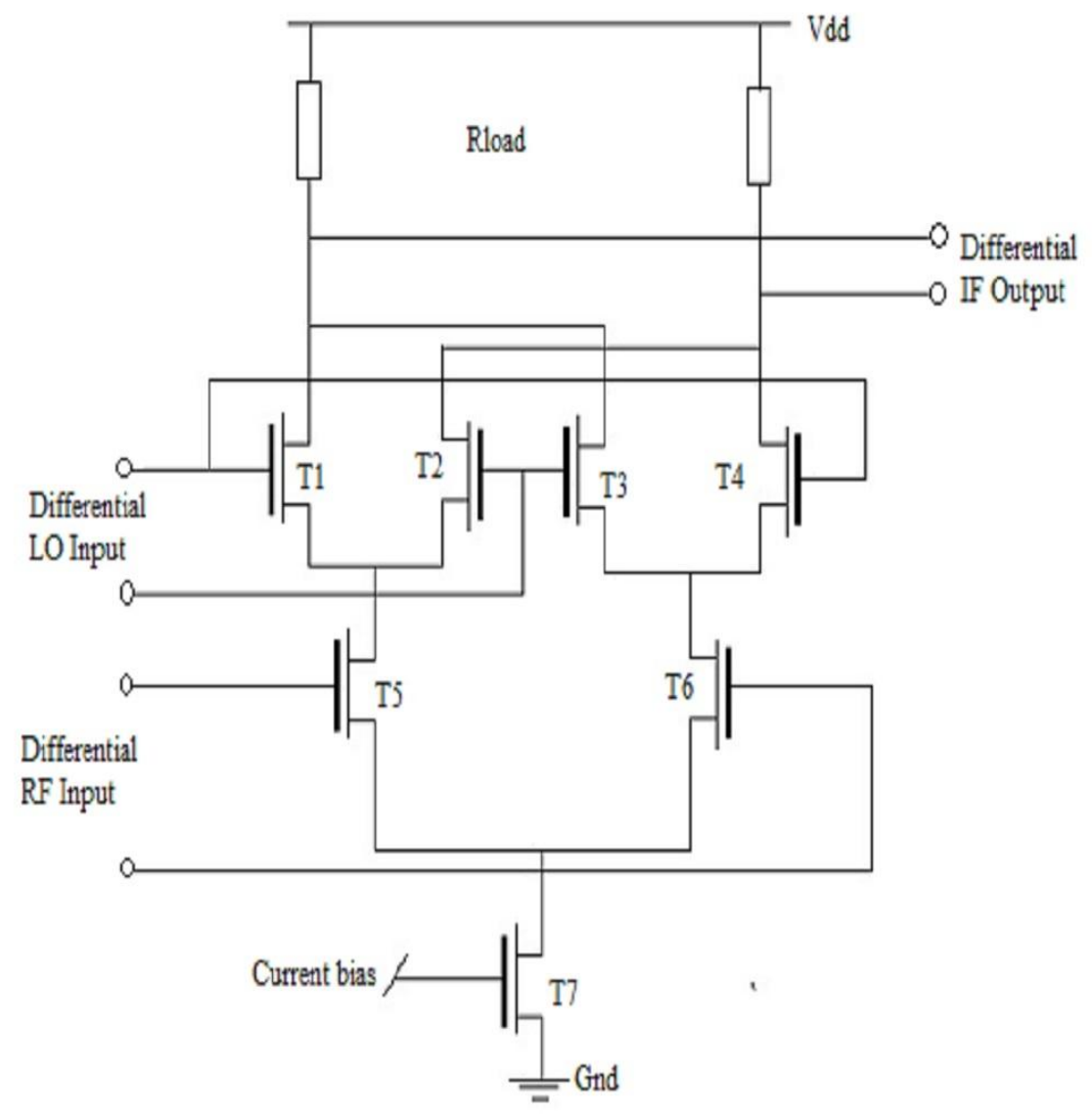

Fig 2: Basic DB Gilbert Cell Mixer

The DB Gilbert cell mixer design equations are given below as shown in [11].

$$
G_{c}=\frac{2}{\pi} R_{L} \sqrt{\mu_{n} C_{O X} \frac{W_{1}}{L_{1}} I_{s s}}
$$

Or if using square wave switching, $\mathrm{CG}$ can be shown as

$$
G_{c}=\frac{2}{\pi} \frac{g_{m} R_{L}}{2}
$$

Port isolation, $\mathrm{P}_{\text {iso }}(\mathrm{L}-\mathrm{R})=\mathrm{P}_{\text {in }}(@ \mathrm{LO})-\mathrm{P}_{\text {out }}(@ \mathrm{RF})$

$$
I I P_{3}=\sqrt{\frac{32}{3} \frac{I_{S S}}{\mu_{n} C_{O X} \frac{W_{1}}{L_{1}}}}
$$

$$
N F=\frac{\pi^{2}}{4}\left(1+\frac{2 \gamma}{g_{m_{-} R F} R_{S}}+\frac{2}{g_{m_{-} R F} R_{S} R_{\text {load }}}\right)
$$

Where $R_{S}$ and $R_{\text {load }}$ are the source and load resistance. The equation consists of the CG, linearity, and NF respectively, while the port isolation is found using the same equation shown in previous page. The simplest harmonic rejection technique is to use band pass filter that passes only the desired band of frequency. In the proposed circuit, the filter is located at the IF port of the mixer. The filter attenuates the harmonics of the mixer.

\subsection{Band Pass Filter}

It allows signals between two specific frequencies to pass, but that discriminates against signals at other frequencies [12]. BPF chebyshev parameter specifications are given below in table 1. 
Table 1. BPF Chebyshev Parameter Specifications

\begin{tabular}{|c|c|c|c|}
\hline Name & Description & Units & Default \\
\hline Fcenter & $\begin{array}{c}\text { Passband center } \\
\text { frequency }\end{array}$ & $\mathrm{MHz}$ & 250 \\
\hline Passband & $\begin{array}{c}\text { Passband edge to } \\
\text { edge width }\end{array}$ & $\mathrm{KHz}$ & 100 \\
\hline Ripple & Passband ripple & $\mathrm{dB}$ & 0.5 \\
\hline Stopband & $\begin{array}{c}\text { Width measured } \\
\text { from lower to } \\
\text { upper stopband } \\
\text { edges }\end{array}$ & $\mathrm{GHz}$ & 1.2 \\
\hline $\mathrm{N}$ & $\begin{array}{c}\text { Filter order(if N }>0, \\
\text { it overwrites } \\
\text { GDpass) }\end{array}$ & None & 3 \\
\hline
\end{tabular}

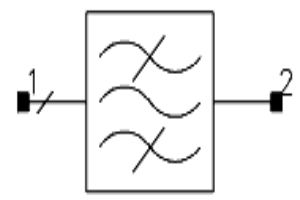

Fig 3: Basic BPF chebyshev filter diagram

Range of usage: BWpass $<$ Fcenter, $0.01 \leq$ Ripple $\leq 3.0,1 \leq$ $\mathrm{N} \leq 15, \mathrm{Qu} \leq 1$

\section{SIMULATIONRESULTS AND DISCUSSION}

Simulations are done over the design implementation of DB Gilbert mixer using ADS Software tools to analyze it performance.The simulation uses the NMOS $180 \mathrm{~nm}$ library.
The MOSFET's that were used in the simulations are the 1.8 volt triple well RF n-channel MOSFET (nMOSFET). The sizes of the devices are varied over wide range of channel length and width, are manipulated to achieve proper functionality. Table 2 specifies the parameters for the mixer design,

Table 2.DBMixer Basic Parameter Specifications

\begin{tabular}{|c|c|}
\hline $\begin{array}{c}\text { Basic Parameter } \\
\text { Specifications }\end{array}$ & Values \\
\hline Biasing Voltage & $1.2 \mathrm{~V}$ to $1.8 \mathrm{~V}$ \\
\hline RF Power & $-50 \mathrm{dBm}$ \\
\hline LO Power & $5 \mathrm{dBm}$ \\
\hline Supply Voltage & $1.8 \mathrm{Volts}$ \\
\hline RF Frequency & $2400 \mathrm{MHz}$ \\
\hline LO Frequency & $2150 \mathrm{MHz}$ \\
\hline IF Frequency & $250 \mathrm{MHz}$ \\
\hline
\end{tabular}

The basic mixer parameter that were modified are displayed in above table affect the mixer design specifications (Conversion Gain, Port Isolation, Harmonic Rejection).

\subsection{DBGilbert Cell Mixer Schematic Diagram and Results}

The fig 4 shows the schematic layout of double balanced Gilbert cell mixer to output an IF frequency of $250 \mathrm{MHz}$. the width specified for local oscillator input transistor is $26 \mu \mathrm{m}$ and for RF input transistor is $231 \mu \mathrm{m}$. Harmonic balance simulations were carried out to study the performance characteristics of the mixer.

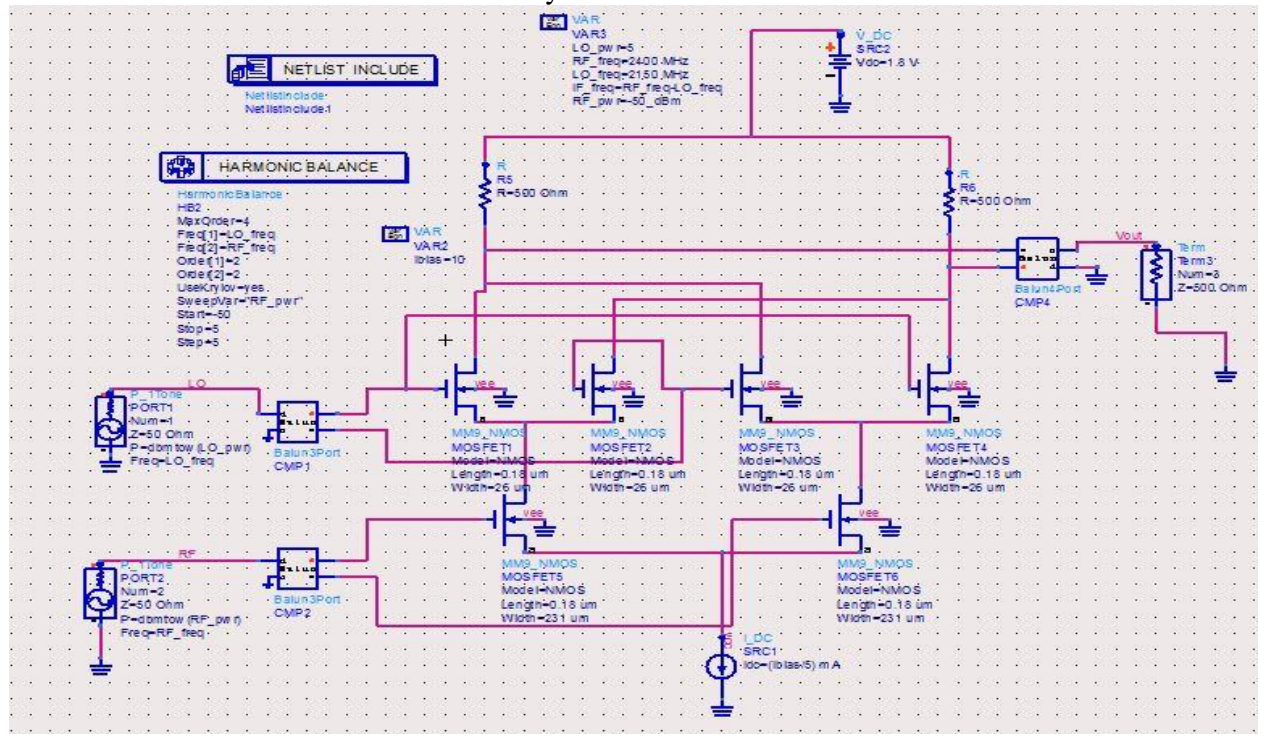

Fig 4:DB Gilbert Cell Mixer Schematic Layout 


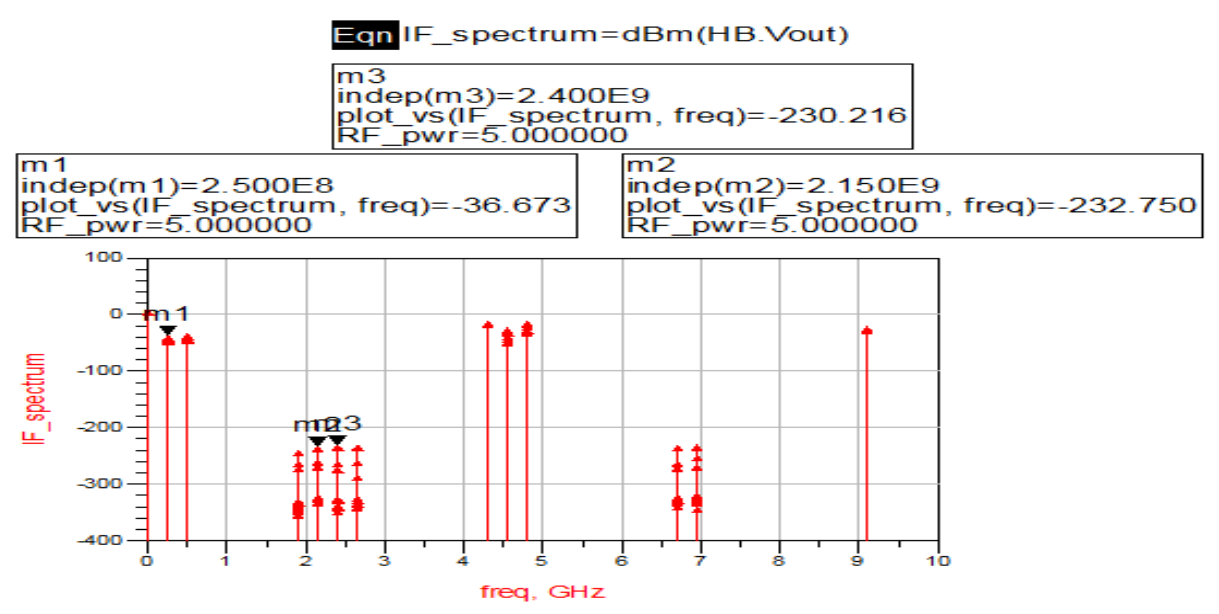

Fig 5:IF Spectrum of DB Gilbert Cell Mixer without Harmonic Rejection

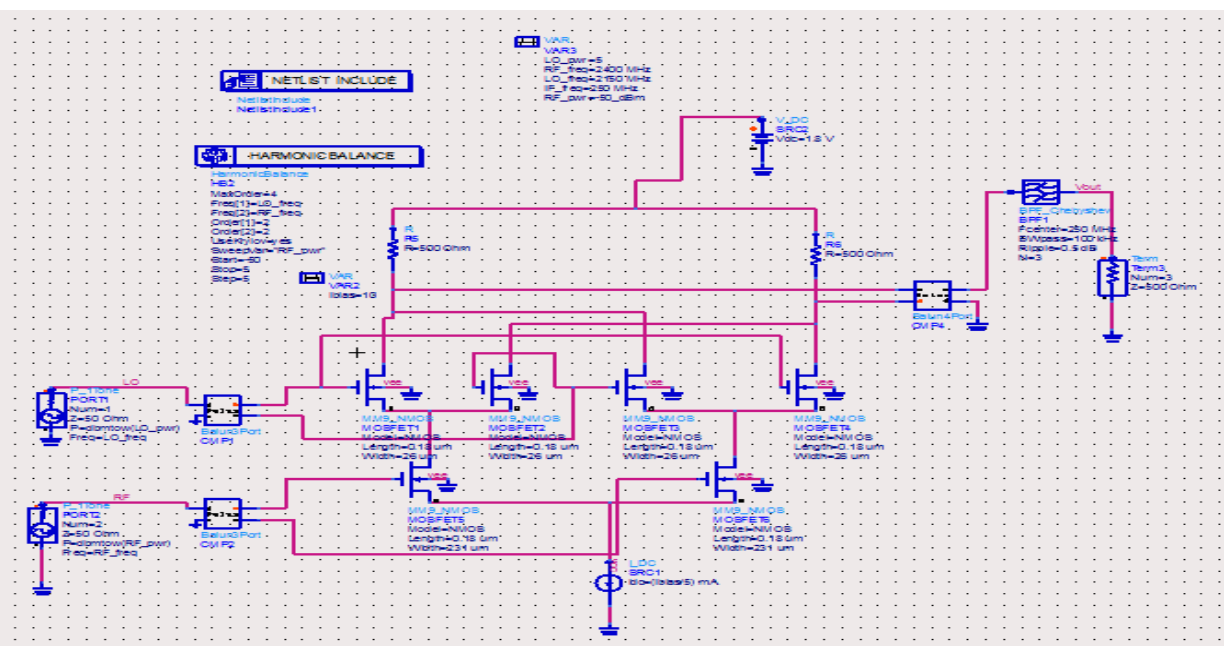

Fig 6:DB Gilbert Cell Mixer Schematic Layout with filter

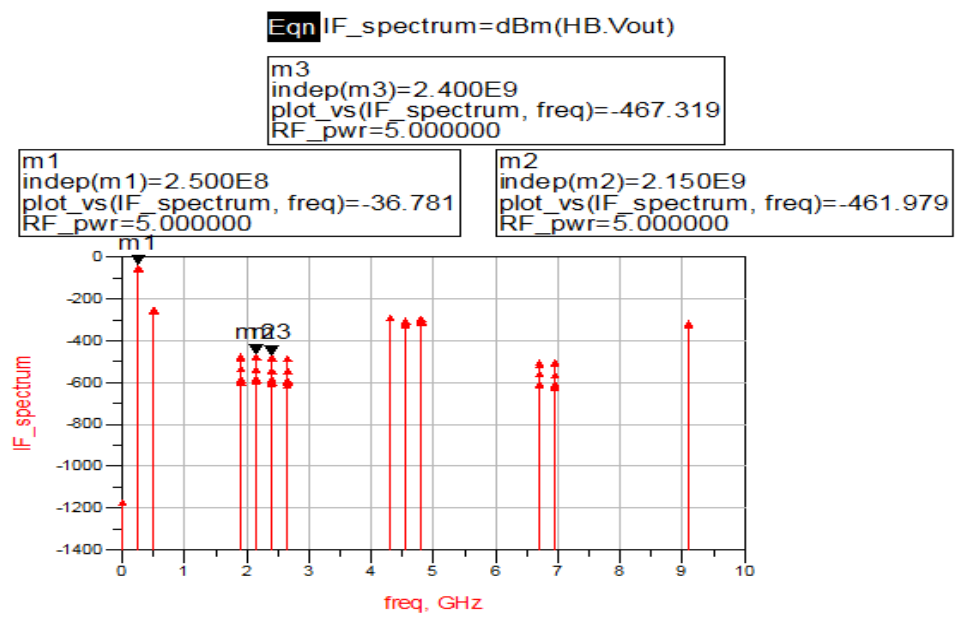

Fig 7: IF Spectrum of DB Gilbert Cell Mixer with filter Harmonic Rejection 
Eqn conveGain=dBm(mix (Vout, $\{1,-1\}))-R F \_p w r$
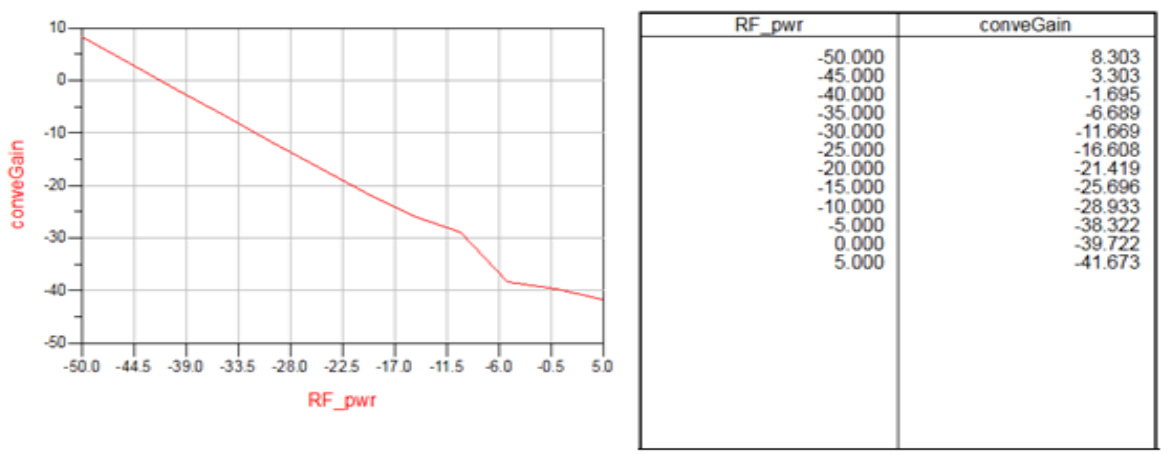

Fig 8: DB Gilbert Cell Mixer Conversion Gain

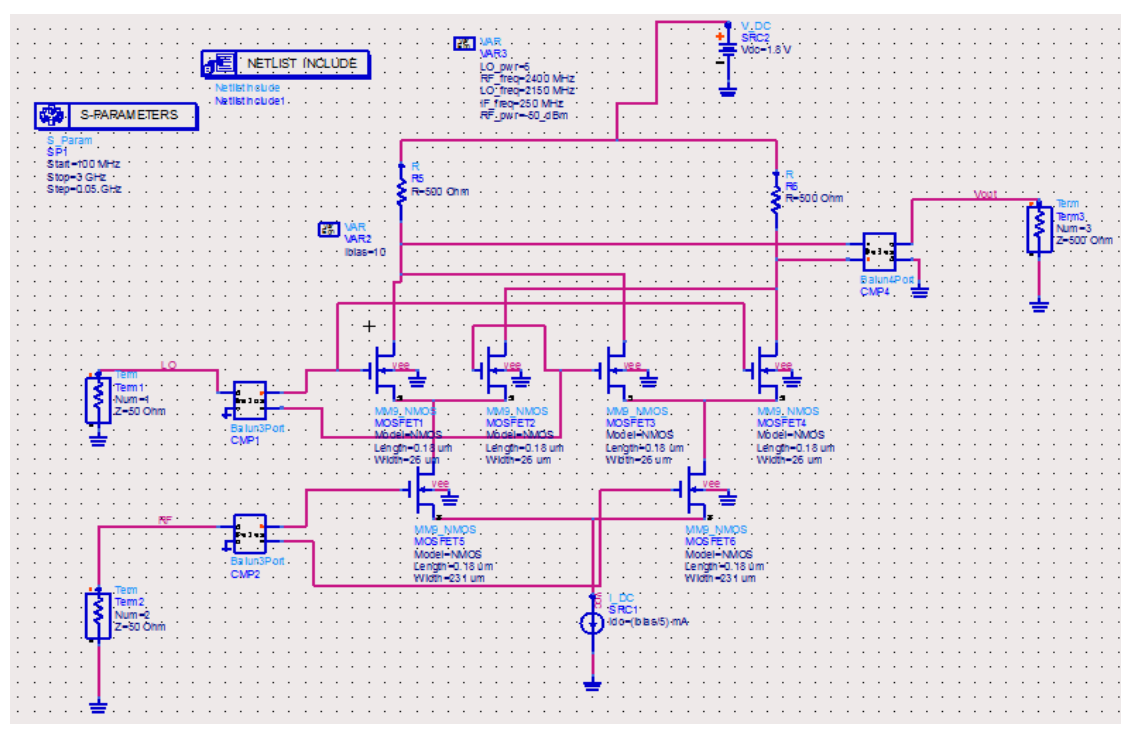

Fig 9:DB Gilbert Cell Mixer without filter Isolation

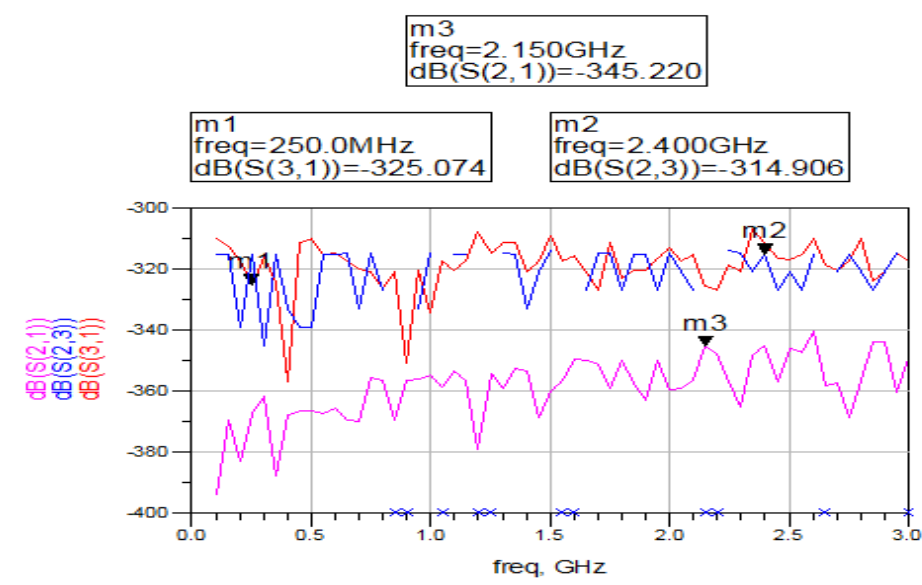

Fig 10: Plot of DB Gilbert Cell Mixer without filter Isolation 


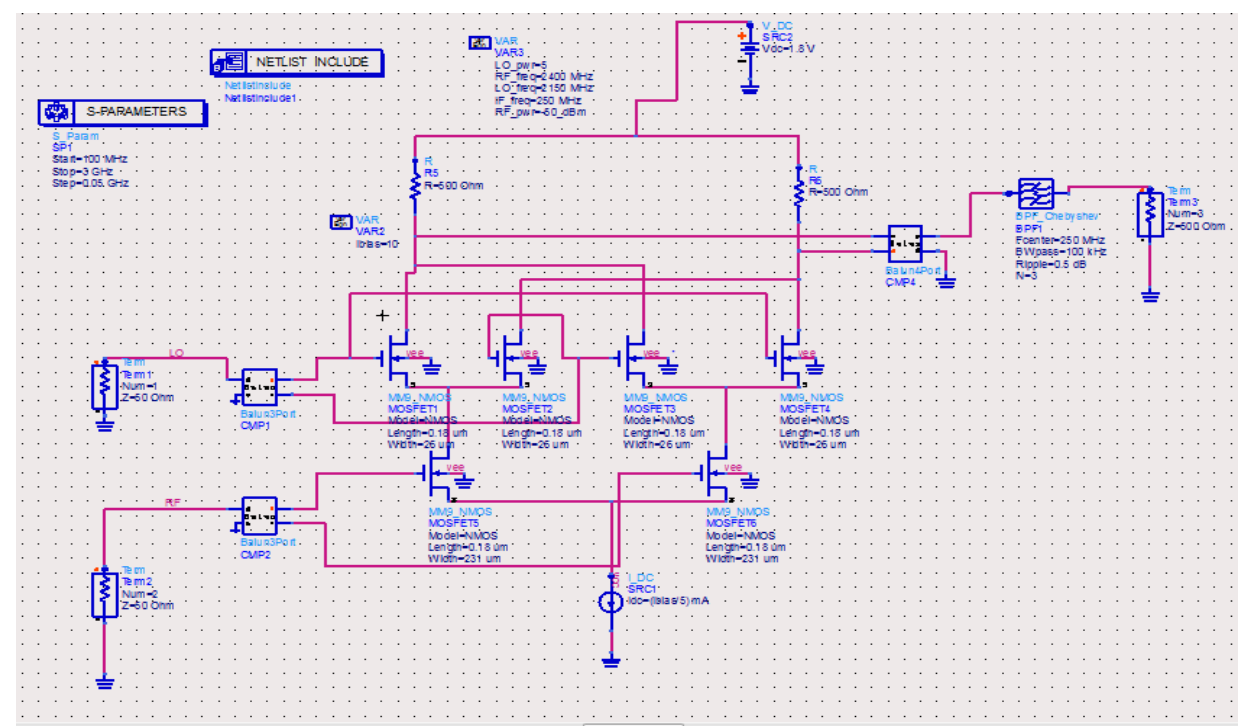

Fig 11: DB Gilbert Cell Mixer with filter Isolation

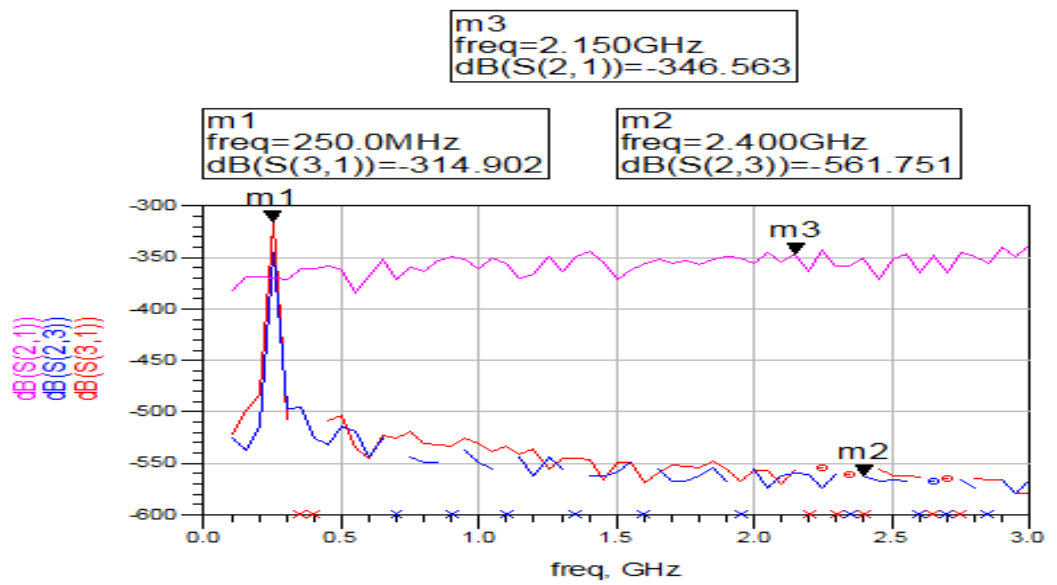

Fig 12: Plot of DB Gilbert Cell Mixer with filter Isolation

The fig 5 represents the IF spectrum of DB Gilbert cell mixer without harmonic rejection techniques. The magnitude of IF spectrum at $250 \mathrm{MHz}$ is $-39.597 \mathrm{dBm}$. The magnitude of third harmonic is around $-200 \mathrm{dBm}$. The fig 6 shows the schematic layout of double balanced Gilbert cell mixer with filter output at IF port to reject the harmonics. Harmonic balance simulation was carried out to study the performance characteristics of the mixer. The fig 7 shows the IF Spectrum of SB Gilbert Cell Mixer with Harmonic Rejection Technique. The magnitude of third harmonics is determined to be $-465 \mathrm{dBm}$. The above fig 8 shows the simulated value of conversion gain in $\mathrm{dBm}$. It is determined to be $8.303 \mathrm{dBm}$. The fig 9 shows the S-parameter simulation of DB gilbert cell mixer without filter to determine port isolation.

In the fig $10, S_{21}$ denotes port isolation between RF port and LO port, $\mathrm{S}_{23}$ denotes port isolation between RF port and IF port, $\mathrm{S}_{31}$ denotes port isolation between IF port and LO port. It is observed that $\mathrm{S}_{21}=-345.220 \mathrm{~dB}, \mathrm{~S}_{23}=-314.906 \mathrm{~dB}, \mathrm{~S}_{31}=-$ $325.074 \mathrm{~dB}$. The port isolation between all the ports in the mixer is less than $-300 \mathrm{~dB}$. Hence the mixer offers good port isolations between each port.The fig 11 shows the $\mathrm{S}$ parameter simulation of DB gilbert cell mixer with filter to determine port isolation. In the fig $12, \mathrm{~S}_{21}$ denotes port isolation between RF port and LO port, $\mathrm{S}_{23}$ denotes port isolation between RF port and IF port, $S_{31}$ denotes port isolation between IF port and LO port. It is observed that S${ }_{21}=-346.563 \mathrm{~dB}, \mathrm{~S}_{23}=-561.751 \mathrm{~dB}, \mathrm{~S}_{31}=-314.902 \mathrm{~dB}$. The port isolation all the ports in the mixer is less than $-300 \mathrm{~dB}$. Hence the mixer offers good port isolations between each port.

\section{CONCLUSION}

In this paper, the CMOS DB Gilbert Cell active mixer with filtering as harmonic rejection technique at IF port is studied, analyzed, and simulated. In the proposed topology, an efficient Chebyshev band pass filter is integrated at the IF port of the downconversion mixer to eliminate the harmonics. DB Gilbert cell mixer with filter at IF port achieves good harmonic rejection ratio $>80 \mathrm{~dB}$ and high port isolation. The work can be further extended by adding polyphase rejection technique in the mixer to reject harmonics.

\section{REFERENCES}

[1] J.P.Silver, "Gilbert Cell Design Tutorial” 2003 http://www.odyseus.nilram.co.uk/RFIC Circuits files / MOS Gilbert Cell Mixer.

[2] Kuei Cheng Lin, Hwann-Kaeo Chiou, et al.., "A 4.2-mW 6-dB Gain 5-65-GHz Gate-Pumped Down Conversion Mixer using Darlington Cell for $60-\mathrm{GHz}$ CMOS Receiver", Microwave Theory and Techniques, IEEE 
Transactions, Vol. 61, Issue4, April 2013, pp.15161522.1

[3] Hwann-Kaeo Chiou, Hung-Ting Chou. “An Ultra-Low Power V-band source -Driven Down Conversion Mixer with Low-Loss and Broadband Asymmetrical BroadsideCoupled Balun in 90-nm CMOS technology", Microwave Theory and Techniques, IEEE Transactions, Vol. 61, Issue 7, July 2013, pp.2620-2631.

[4] Ning Zhang, HaifengXu, et al.., "W-Band Active DownConversion Mixer in Bulk CMOS". Microwave and Wireless Components Letters, IEEE Microwave theory and techniques society, Vol. 19, Issue 2, February 2009, pp.98-100.

[5] Aslam A. Rafi, T.R Viswanathan. "Harmonic Rejection Mixing Techniques using Clock-Gating". Solid State Circuits, IEEE Journals, Vol. 48, Issue 8, August 2013, pp. 1862-1874.

[6] Fujian Lin, Pui-In Mak et al.., "A Sine LO Square Law Harmonic Rejection Mixer Theory" Microwave Theory and Techniques, IEEE Transactions, Vol. 62, Issue 2,February 2014, pp.313-322.

[7] Renzhi Liu, Pileggi L et al..,"A Wideband RF Receiver with >80 dB Harmonic Rejection Ratio". Custom
Integrated Circuits Conference (CICC), IEEE, September 2014, pp.1-4.

[8] Huajiang Zhang, Tian Bao Gao, et al.., "Harmonic Rejection Mixer with Improved Design Algorithm for Broadband TV Tuners". Radio Frequency Integrated Circuits Symposium (RFIC),IEEE, June 2012, pp. 163166.

[9] Takafumi Yamaji Tanimoto H, et al.., "Harmonic Signal Rejection Schemes of Poly phase Down Converters". Circuits and Systems I:Regular Papers, IEEE Transactions, Vol. 58, Issue 10, October 2011, pp.23082317.

[10] L.A NacEachern, Y. Manku, "A Charge-Injection Method for Gilbert Cell Biasing",in Electrical and Computer Engineering Canadian Conference, 1998, pp.365-368.

[11] World Academy of Science Engineering and Technology, "A Low Voltage High Linearity CMOS Gilbert Cell using Charge Injection Method,"2008, http://www.waset.org

[12] http://whatis.techtarget.com/definition/bandpass-filter. 